\title{
Altered Frequency of NK Cells and Treg Cells by Astragalus Polysaccharide Combined with Budesonide in Asthma Model Mice
}

\author{
Wei Zhang $\mathbb{D}^{1}$ and Kuifen Ma $\mathbb{D}^{2}$ \\ ${ }^{1}$ Department of Respiratory Medicine, Affiliated Hangzhou First People’s Hospital, Zhejiang University School of Medicine, \\ Hangzhou 310006, China \\ ${ }^{2}$ The First Affiliated Hospital of Medical School of Zhejiang University, Hangzhou 310003, China
}

Correspondence should be addressed to Wei Zhang; bobbyzw@163.com

Received 21 August 2019; Accepted 28 October 2019; Published 12 January 2020

Guest Editor: Jianxun Ding

Copyright (C) 2020 Wei Zhang and Kuifen Ma. This is an open access article distributed under the Creative Commons Attribution License, which permits unrestricted use, distribution, and reproduction in any medium, provided the original work is properly cited.

\begin{abstract}
Objective. We investigated the efficacy of astragalus polysaccharide (APS) combined with budesonide and the effect on expressions of peripheral NK cells and Treg cells and the molecular mechanism in mice with bronchial asthma. Methods. In this study, we established a mouse model of asthma. Four groups of $\mathrm{BaLB} / \mathrm{C}$ mice were developed; control group had no asthma induction, and the other three groups of mice were sensitized by OVA (Ovalbumin), OVA + budesonide, and OVA + APS + budesonide. Flow cytometry was used to determine the proportion of NK cells and Treg cells. Levels of cytokines IL- 4 and IL-10 were detected using RT-PCR and ELISA. Results. Asthma mice treated with APS + budesonide showed alleviated airway resistance compared to model mice $(P<0.05)$. The percentage of dendritic cells (DCs) was reduced $(P<0.05)$, while anti-inflammatory NK cells and Treg cells significantly increased after APS + budesonide treatment $(P<0.05)$. Further, APS + budesonide treatment resulted in improvements in IL-4 and IL-10 mRNA and protein levels $(P<0.05)$. Conclusion. APS combined with budesonide medication may regulate expressions of DCs cells and related cytokines, reliving clinical symptom of bronchial asthma.
\end{abstract}

\section{Introduction}

Bronchial asthma is a common chronic airway inflammatory disease, and its pathogenesis includes a variety of cells and components. Based on epidemiological survey data, elderly people have a higher incidence of asthma [1]. At present, the main treatment is drug therapy, which can be classified as control patient's condition and relief symptoms. Long-term oral or intravenous use of glucocorticoid can cause immunosuppression and Cushing's syndrome and other side effects. Inhalation of common doses of glucocorticoid generally does not cause adverse reactions [2]. But after glucocorticoids inhalation, approximately $80-90 \%$ of the drug is deposited in the pharynx and swallowed into the gastrointestinal tract, eventually depositing glucocorticoid will cause pharyngeal or systemic adverse reactions.

Over the last decades, traditional Chinese medicine extracted from the root of Astragalus membranaceus was documented to have biological activities. Astragalus polysaccharide (APS), the extraction, is a polysaccharide consisting of glucose and arabinose sugar, possessing very low toxicity, with a wide range of immune enhancement and antiviral effect, which can relieve the plant hemagglutination (PHA) toxicity and resist the inhibitory effects of immunosuppressive drugs such as prednisolone and cyclophosphamide [3]. The therapeutic implication of APS has been confirmed in patients with lung cancer [4]. Budesonide, which is a commonly used inhaled hormone, has fewer systemic adverse reactions. However, there are a few clinical studies on the combined therapy for adult asthma.

Given the NK cells and regulatory $\mathrm{T}$ cells contribute to asthma pathogenesis, it is believed that combination of APS and budesonide may influence immune cell counts. In this study, the effects of APS combined with budesonide on pulmonary function and immune response in mice with bronchial asthma were observed, aiming to provide theoretical basis for clinical treatment. 


\section{Methods and Materials}

2.1. Animals and Study Design. Forty female BaLB/c mice $(8.7 \pm 3.4 \mathrm{~g})$ purchased from Shanghai Lab Animal Research Center (Shanghai, China) were divided into 4 groups: control group $(n=8)$, untreated asthma group $(n=8)$, budesonide group $(n=8)$ and APS + budesonide group $(n=8)$. Mouse model of asthma were induced as previously [5]. Briefly, $25 \mu \mathrm{g}$ OVA (SIGMA-ALDRICH, O1641) dissolved in $200 \mu \mathrm{L}$ PBS were injected intraperitoneally into the mice except those in control group. Mice in the control group were injected with same volume of PBS. On day 14, mice in the non-control group were kept in a closed container and inhaled 6\% OVA solution by ultrasonic atomization to induce asthma $30 \mathrm{~min} /$ day for 7 days while mice in the control group inhaled PBS. On day 15 , control and untreated asthma group was administered with atomized PBS; budesonide group, $3 \mu \mathrm{g} /$ day budesonide; APS + budesonide group, $100 \mathrm{mg} / \mathrm{kg}$ body weight/day APS injected intraperitoneally and $3 \mu \mathrm{g} /$ day budesonide inhaled. On day 29, mice were sacrificed to evaluate airway response and other biomarkers.

2.2. Bone-Marrow-Derived DCs (BMDCs) Generation. The tibial and femoral bone marrow was isolated from the mice and the BMDCs were prepared as previous reported [6]. Isolated BMDCs were cultured in RPMI-1640 containing $5 \%$ fetal bovine serum and stimulated with $1000 \mathrm{U} / \mathrm{mL}$ IL-4 and $500 \mathrm{U} / \mathrm{mL}$ granulocyte-macrophage colony-stimulating factor (GM-CSF). On day 7, non-adherent cells were harvested.

2.3. Flow Cytometric Analysis of NK, DCs and Treg Cells. A FC500 (Beckman Coulter, USA) was used to analyze the proportions of NK, DCs and Treg cells. The data was analyzed with CXP analysis software 2.3. NK cells were stained with FITC-CD16 and PE-CD56 (eBioscience, SanDiego, CA). The BMDCs were stained with rat anti-mouse monoclonal antibodies to FITC-CD11c and PE-CD86 (B7-2) (eBioscience, SanDiego, CA). Treg cells were stained with FITC-CD4 and PE-CD25 (eBioscience, SanDiego, CA).

2.4. Determination of Airway Hyper-Responsiveness. Airway hyper-responsiveness was measured using the FinePointe ${ }^{\mathrm{Tm}} \mathrm{RC}$ animal airway resistance and pulmonary compliance system (BUXCO, USA). On day $28,60 \mathrm{mg} / \mathrm{kg}$ pentobarbital sodium was injected intraperitoneally, then a tracheal incision was performed. Respiratory frequency was set at 160 times/min. Mice were inhaled with $10 \mu \mathrm{L}$ acetylcholine, at ascending concentrations of $0 \mathrm{mg} / \mathrm{mL}, 1 \mathrm{mg} / \mathrm{mL}, 2 \mathrm{mg} / \mathrm{mL}, 4 \mathrm{mg} / \mathrm{mL}$, $8 \mathrm{mg} / \mathrm{mL}$. The maximum airway resistance within 3 minutes was recorded.

2.5. Quantitative RT-PCR. Total RNA from the lung tissue was extracted using Trizol reagent (Takara, Dalian, China), and cDNA was synthesized using the Prime Script ${ }^{\oplus}$ RT reagent kit (Takara, Dalian, China), according to the manufacturer's instructions. Real-time RT-PCR was performed using SYBR ${ }^{\oplus}$ Premix Ex TapTM II (Takara). The primers used are as follows: IL4 (120 bp), forward prime, $5^{\prime}$-CATCGGCATTTTGAACGAG-3' , reverse prime, 5' -TTGGAAGCCCTACAGACGAG-3'; IL10 (165bp), forward prime, $5^{\prime}$-TAACTGCACCCACTTCCCAG-3', reverse prime, $5^{\prime}$-AAGGCTTGGCAACCCAAGTA-3'; GAPDH (104bp), forward prime, $5^{\prime}$-CGTGTTCCTACCCCCAATGT-3', reverse prime, 5'-TGTCATACTTGGCAGGTTTCT-3'. All experiments were conducted in triplicate and repeated three times. The PCR data were analyzed using the QuantStudio 7 Flex detection system (Applied Biosystems Co., Foster City, CA, USA). Cycle threshold (CT) values were calculated using the comparative CT $(\Delta \Delta \mathrm{CT})$ method, and the relative amount of target mRNA $\left(2^{-\Delta \Delta C T}\right)$ was obtained by normalizing to endogenous GAPDH levels.

2.6. ELISA Assay. Bronchoalveolar lavage fluid (BALF) was collected and analyzed for interleukin-4 (IL-4) and IL-10 contents by enzyme-linked immunosorbent assay (ELISA), according to the manufacturer's guidelines.

2.7. Statistical Analyses. All data were analyzed using SPSS13.0 software. The data are expressed as mean \pm standard deviation. The inter-group comparisons of means were performed using univariate analysis of variance and pairwise comparison between groups, with $P<0.05, P<0.01$ or $P<0.001$ as significantly different.

\section{Results}

3.1. Body Weight and Airway Resistance in Mice with Asthma Influences by APS and Budesonide. Compared to control group, mice in model group, and drug treated group had a loss and slight fluctuation in body weight (Figure 1(a)). Airway hyper-responsiveness was assessed in 4 groups. In untreated group, mice exposed to OVA showed significant airway resistance at acetylcholine concentrations of 1,2, 4, and $8 \mathrm{mg} / \mathrm{mL}(P<0.01$; Figure $1(\mathrm{~b}))$. To verify airway resistance, the airway resistance after acetylcholine challenge between untreated model mice and various treatment groups was compared. The mice of three various treated groups exhibited significantly less airway resistance at an acetylcholine concentration of $1,2,4$, and $8 \mathrm{mg} / \mathrm{mL}(P<0.05$; Figure $1(\mathrm{~b}))$. The mice in APS + budesonide showed significantly lower airway resistance than that in budesonide group $(P<0.05$; Figure 1(b)).

\subsection{Percentage of DCs, NK Cells, and Treg Cells Influences by} APS and Budesonide. The frequency of NK cells and Treg cells in BALF has been analyzed. Compared with healthy controls, animals with experimental asthma showed attenuated DCs and attenuated cell numbers of NK cells and Treg cells $(P<0.05$; Figure 2). However, the percentages of NK cells and Treg cells, were markedly increased in mice with drug treatment whether combined with APS $(P<0.05$; Figure 2$)$. The frequency of DCs in untreated asthma group was elevated when compared to control group $(P<0.05$; Figure 2). By comparison, frequency of DCs in two treated groups was significantly alleviated $(P<0.05$; Figure 2$)$. In addition, the 


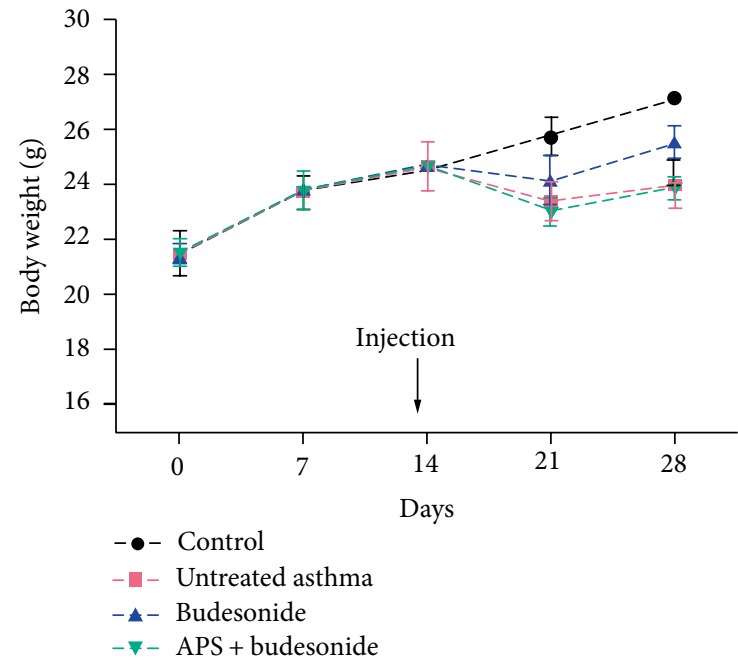

(a)

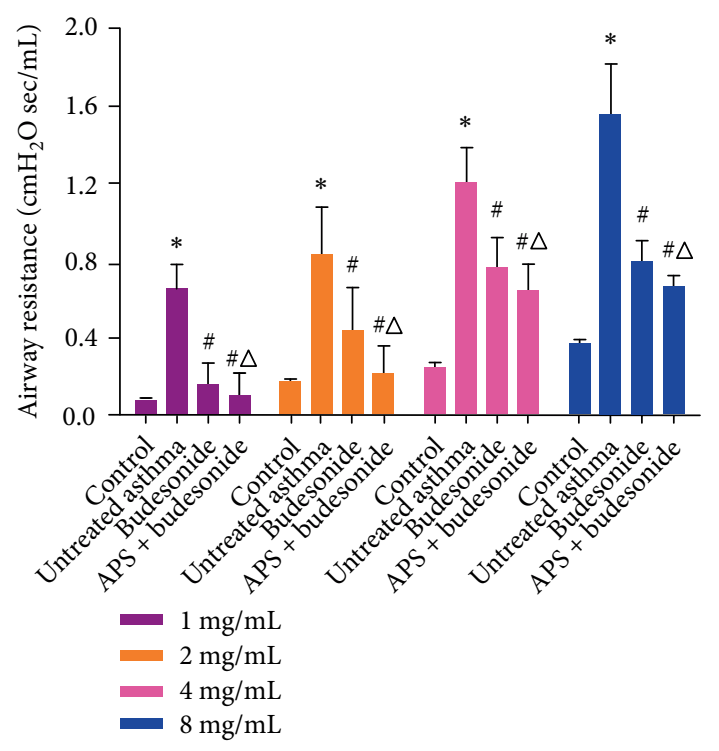

(b)

FIGURE 1: Body weight and airway resistance effected by APS and budesonide treatment. (a) The body weight change of mice in 4 groups $(n=8$ per group) was monitored within 28 days. (b) Airway resistance in mice was measured after treatment. Mean \pm SD is presented for each group. ${ }^{*} P<0.01$, compared with control group; ${ }^{\sharp} P<0.05$, compared with untreated asthma group; ${ }^{\wedge}<<0.05$, compared with budesonide group.

frequency of DCs in APS + budesonide group was significantly lower than that in budesonide group $(P<0.05$; Figure 2$)$.

\section{3. mRNA Levels of IL4 and IL10 in Mice Influences by APS and}

Budesonide. As NK cells in the lungs have been considered to be regulators of Th1 or Th2 cytokine production, we analyzed the mRNA levels of inflammatory mediators: IL4 and IL10 in lung tissues of mice. Th2 cytokines IL 4 and anti-inflammatory cytokines IL10 in mice model of asthma were significantly increased, compared to control group $(P<0.05$; Figure 3$)$. Administration of budesonide significantly reduced the mRNA levels of IL4 in mice with asthma $(P<0.05$; Figure 3$)$. Levels of IL4 and IL10 in APS + budesonide group were significantly decreased compared to budesonide group $(P<0.05$; Figure 3$)$.

3.4. Cytokine Levels in APS and Budesonide Treated Mice. Levels of the important Th2 cytokine IL- 4 and inflammatory cytokine IL-10 in BALF and the spleen were measured by ELISA. As shown in Figure 4, levels of these cytokines in BALF significantly declined in APS + budesonide-treated mice, when compared with budesonide treated mice $(P<0.05$; Figure 4). Levels of IL-4 and IL-10 in spleen were consistent with that in BALF.

\section{Discussion}

The etiology of asthma is complex, and airway hyperresponsiveness is the characteristic pathological manifestation of asthma. Leukotriene, a metabolite of arachidonic acid, is widely present in the airway of patients with bronchial asthma and mediates and induces inflammatory reactions. Previous clinical practice has shown that the clinical effect of montelukast alone as a leukotriene regulator in the treatment of bronchial asthma needs to be further improved. Polysaccharide served as anti-tumor drug has been well studied in various types of tumor cells $[7,8]$. In the present study, we established a murine asthmatic model and demonstrated that asthma model mice, challenged with APS and budesonide, exhibited improved airway hyper-responsiveness.

Current studies have confirmed that the pathogenesis of bronchial asthma is closely related to cellular immune dysfunction. The cell types involved in the pathological process of asthma include Th 2 cells, Th17 cells, NK cells, antigen presenting cells, B cells, etc. [9-13]. As an important natural immune cell, NK cells are the third type of lymphocytes along with T cells and B cells. Recent studies have shown that compared with healthy controls, NK cells isolated from the peripheral blood of asthmatic patients have a reduced killing ability against tumor cell lines, although they secrete more cytotoxic regulators [14]. The airway inflammatory immune response of bronchial asthma is associated with decreased cytotoxicity of NK cells and increased leukocyte count. Some studies have confirmed that NK cell subsets are significantly reduced in patients with allergic asthma, suggesting that the occurrence of asthma is related to the inhibition of NK function [15]. Moreover, the NK cells content in the remission phase of asthma was higher than that in the acute attack phase $[16,17]$. NK cells is a necessary factor for naive T cells differentiating to Th2 cells.

Treg plays an important role in balancing the inflammatory response and regulating the immune response. Studies have found that in allergic patients, Treg content is reduced and function is impaired. In addition, Treg cells can negatively feedback regulate $T$ cells through a variety of inhibitory mechanisms, such as down-regulating the expression of T cytokines and inhibiting the activation and differentiation of T cells [18]. DCs can be directly activated and indirectly activated by 

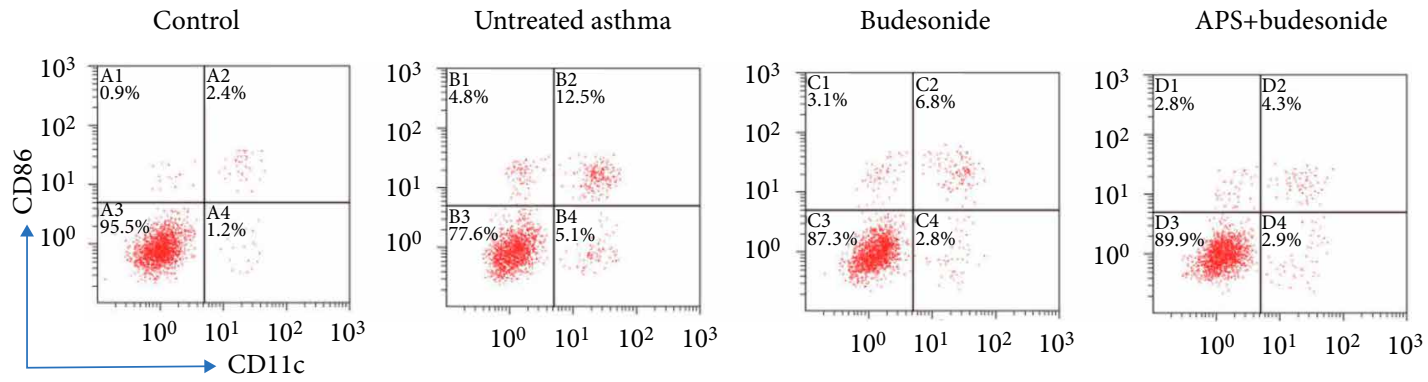

(a)
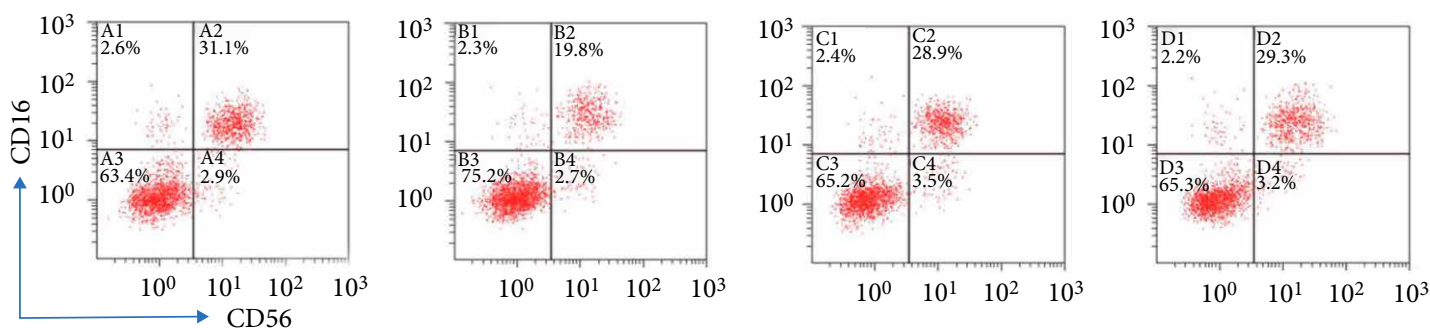

(b)
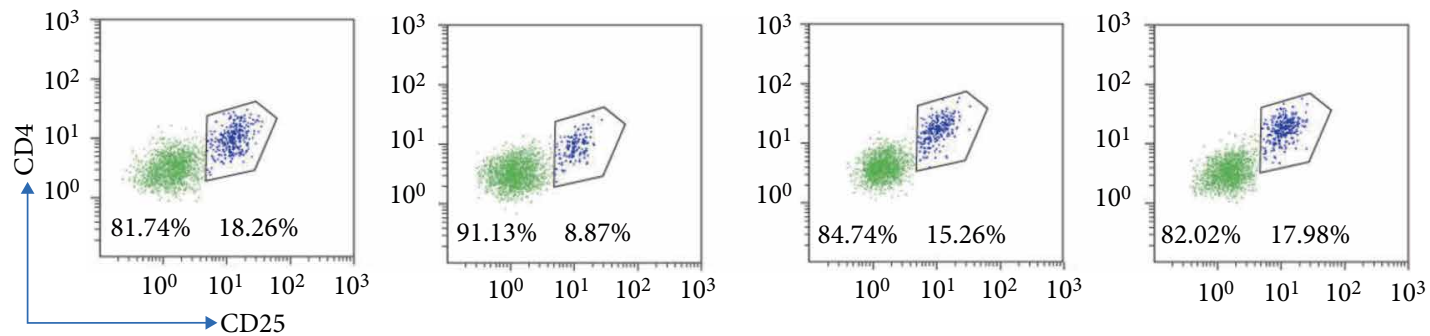

(c)

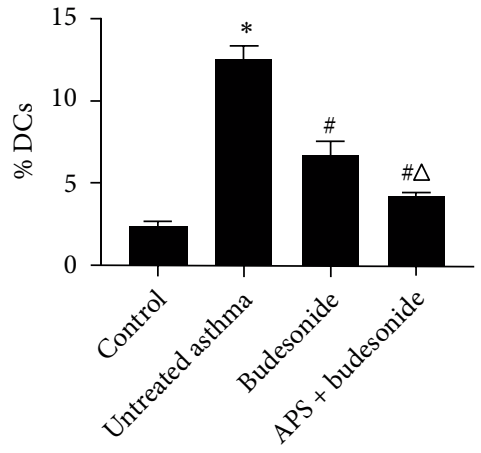

(d)

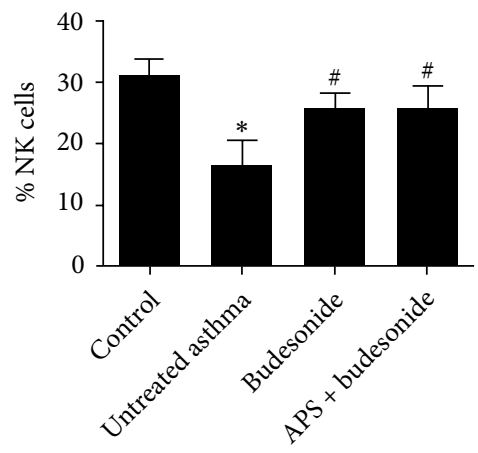

(e)

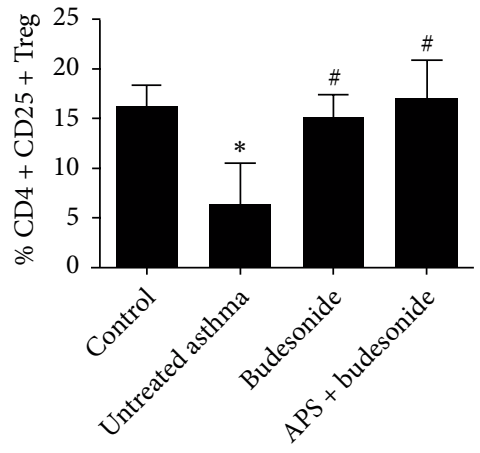

(f)

FIGURE 2: Flow cytometric analysis of DCs, NK cells, and Treg cells. (a-c) The percentage of DCs, NK cells, and Treg cells. (d-f) Results from gated cells were visualized by bar charts as percentages of DCs, NK cells, and Treg cells. Mean \pm SD is presented for each group. ${ }^{*} P<0.01$, compared with control group; ${ }^{\#} P<0.05$, compared with untreated asthma group; ${ }^{\triangle} P<0.05$, compared with budesonide group.

epithelium when inhaling harmless allergens, which are sufficient to initiate Th2 immunity. Our results shed light upon how the polysaccharide influence the asthma model mice. Using the OVA-induced asthma model, our data showed that combination of APS and budesonide modulated the proportion of DCs, NK cells, and Treg cells. Notably, APS and budesonide had significant decrease of DCs compared to budesonide alone, suggesting the reliving immune response in treated-asthma mice.

It is documented that patients with asthma often had increased frequencies of IL-4-producing NK cells [19].
Impaired capacity of DCs to activate T cells results in incomplete maturation of specific inhibitory cytokines such as IL-10, which will suppress T-cell immune reactivity [20-22]. In this study, we observed the mRNA levels of IL-4 and IL-10 were alleviated by APS and budesonide treatment. Furthermore, both local (BALF) and systemic (spleen) levels of IL-4 and IL-10 in mice treated with APS and budesonide were significantly lower than in untreated model mice. Therefore, it is possible that APS and budesonide suppressed the production of IL-4 and IL-10 in gene and protein levels. 


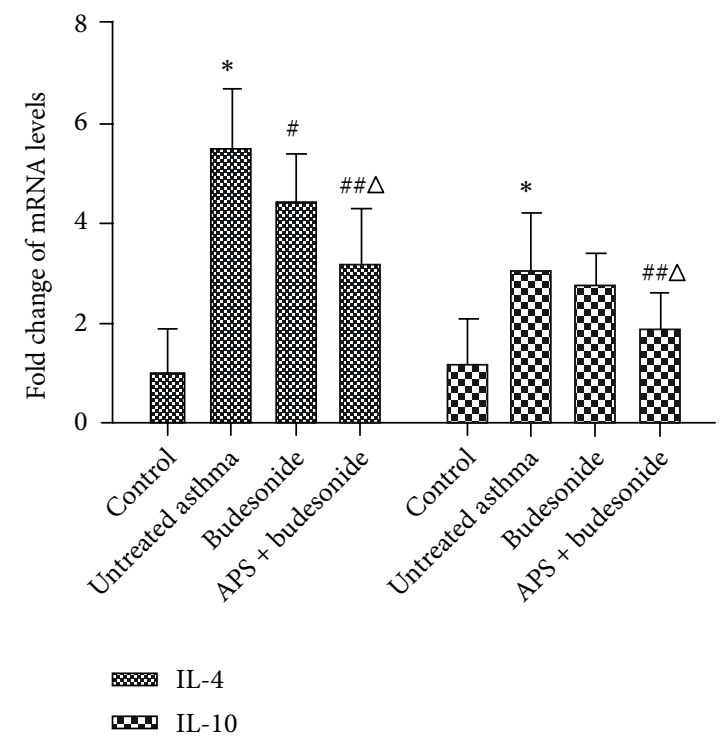

FIGURE 3: APS and budesonide suppressed mRNA levels of inflammatory mediators. Relative expression of IL4 and IL10 mRNA levels were measured by RT-PCR and is represented above. Every independent experiment was triplicated. Mean \pm SD is presented for each group. ${ }^{*} P<0.01$, compared with control group; ${ }^{\#} P<0.05$, compared with untreated asthma group; ${ }^{\wedge} P<0.05$, compared with budesonide group.

BALF

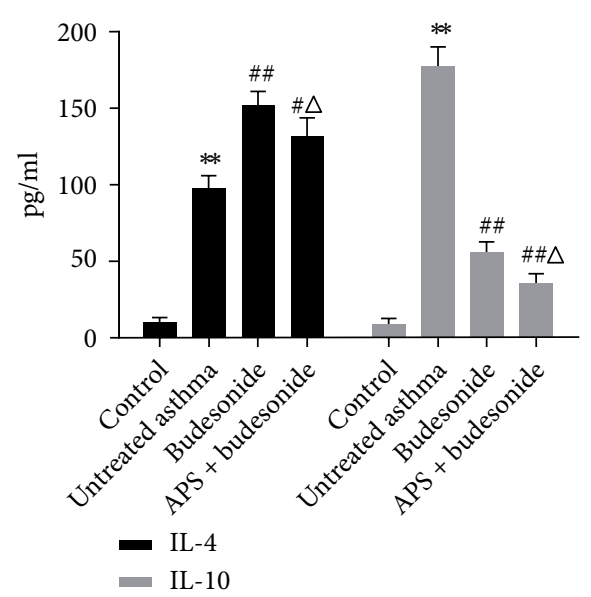

Spleen

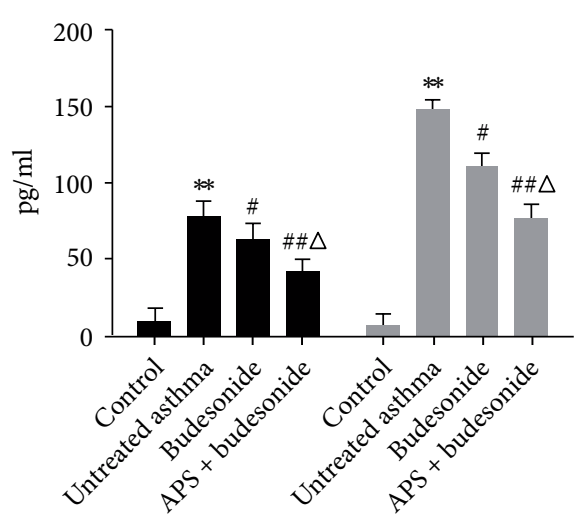

FIGURE 4: Cytokine levels of IL-4 and IL-10 in BALF and spleen were analyzed by ELISA. Every independent experiment was repeated twice. Mean \pm SD is presented for each group. ${ }^{* *} P<0.01$, compared with control group; ${ }^{\#} P<0.05,{ }^{\# \#} P<0.01$, compared with untreated asthma group; ${ }^{\triangle} P<0.05$, compared with budesonide group.

Taken together, these findings suggest that budesonide with or without APS treatment might induce protective immunity against asthma by the regulation of DCs, NK cells and Treg cells through IL- 4 and IL-10 production. Apart from that, combination of APS and budesonide shows benefits in improving airway pressure maybe due to the modulation of DCs, IL-4 and IL-10, which provide a new strategy in the therapy of asthma.

\section{Data Availability}

The data used to support the findings of this study are available from the corresponding author upon request.

\section{Conflicts of Interest}

The authors declare that there are no conflicts of interest regarding the publication of this paper.

\section{Acknowledgments}

This work was supported by Zhejiang Provincial Health Department Project (Grant no. 2019KY486).

\section{References}

[1] I. Kuprys-Lipinska, J. Liebhart, C. Palczynski, P. Lacwik, M. Jonakowski, and P. Kuna, "Prevalence, risk factors and 
underdiagnosis of asthma in the general population aged over 60 years," Postepy Dermatologii I Alergologii, vol. 36, no. 1, pp. 86-91, 2019.

[2] P. Nair and P. Barker, "Glucocorticoid sparing of benralizumab in asthma," The New England Journal of Medicine, vol. 377, no. 12, p. 1205, 2017.

[3] K. K. Auyeung and Q. B. Han, “Astragalus membranaceus: a review of its protection against inflammation and gastrointestinal cancers," The American Journal of Chinese Medicine, vol. 44, no. 1, pp. 1-22, 2016.

[4] O. A. Bamodu, K. T. Kuo, C. H. Wang et al., "Astragalus polysaccharides (PG2) enhances the M1 polarization of macrophages, functional maturation of dendritic cells, and T cell-mediated anticancer immune responses in patients with lung cancer," Nutrients, vol. 11, no. 10, p. 2264, 2019.

[5] S. Wu, G. Wang, and R. Yang, "Anti-inflammatory effects of polysaccharide on asthma pathology," American Journal of Translational Research, vol. 8, no. 10, pp. 4478-4489, 2016.

[6] K. J. Liu, S. J. Leu, C. H. Su, B. L. Chiang, Y. L. Chen, and Y. L. Lee, "Administration of polysaccharides from Antrodiacamphorata modulates dendritic cell function and alleviates allergeninduced T helper type 2 responses in a mouse model of asthma," Immunology, vol. 129, no. 3, pp. 351-362, 2010.

[7] H. Guo, F. Li, W. Xu et al., "Mucoadhesive cationic polypeptide nanogel with enhanced penetration for efficient intravesical chemotherapy of bladder cancer," Advanced Science (Weinheims), vol. 5, no. 6, p. 1800004, 2018.

[8] J. Wang, W. Xu, S. Li et al., "Polylactide-cholesterol stereocomplex micelle encapsulating chemotherapeutic agent for improved antitumor efficacy and safety," Journal of Biomedical Nanotechnology, vol. 14, no. 12, pp. 2102-2113, 2018.

[9] Y. M. Du, Y. X. Zhuansun, R. Chen, L. Lin, Y. Lin, and J. G. Li, "Mesenchymal stem cell exosomes promote immunosuppression of regulatory T cells in asthma," Experimental Cell Research, vol. 363, no. 1, pp. 114-120, 2018.

[10] P. J. Barnes, "Targeting cytokines to treat asthma and chronic obstructive pulmonary disease," Nature Reviews Immunology, vol. 18, no. 7, pp. 454-466, 2018.

[11] M. C. Altman, E. Whalen, A. Togias et al., "Allergen-induced activation of natural killer cells represents an early-life immune response in the development of allergic asthma," The Journal of Allergy and Clinical Immunology, vol. 142, no. 6, pp. 1856-1866, 2018.

[12] D. C. Do, Y. Zhao, and P. Gao, "Cockroach allergen exposure and risk of asthma," Allergy, vol. 71, no. 4, pp. 463-474, 2016.

[13] M. H. Grayson, S. Feldman, B. T. Prince, P. J. Patel, E. C. Matsui, and A. J. Apter, "Advances in asthma in 2017: mechanisms, biologics, and genetics," Journal of Allergy and Clinical Immunology, vol. 142, no. 5, pp. 1423-1436, 2018.

[14] M. G. Duvall, C. Barnig, M. Cernadas et al., "Natural killer cellmediated inflammation resolution is disabled in severe asthma," Science Immunology, vol. 2, no. 9, , 2017.

[15] B. Simons, M. E. Ferrini, S. Carvalho, D. J. P. Bassett, Z. Jaffar, and K. Roberts, "PGI2 controls pulmonary NK cells that prevent airway sensitization to house dust mite allergen," Journal of Immunology, vol. 198, no. 1, pp. 461-471, 2017.

[16] J. H. Kim and Y. J. Jang, "Role of natural killer cells in airway inflammation," Allergy Asthma \& Immunology Research, vol. 10, no. 5, pp. 448-456, 2018.
[17] M. M. Gorska, "Natural killer cells in asthma," Current Opinion in Allergy and Clinical Immunology, vol. 17, no. 1, pp. 50-54, 2017.

[18] D. M. Richards, M. Delacher, Y. Goldfarb et al., "Treg cell differentiation: from thymus to peripheral tissue," Progress in Molecular Biology and Translational Science, vol. 136, pp. 175-205, 2015.

[19] A. K. Singh, S. Rhost, L. Löfbom, and S. L. Cardell, "Defining a novel subset of CD1d-dependent type II natural killer T cells using natural killer cell-associated markers," Scandinavian Journal of Immunology, vol. 90, no. 3, p. e12794, 2019.

[20] K. J. Liu, S. J. Leu, C. H. Su, B. L. Chiang, Y. L. Chen, and Y. L. Lee, "Administration of polysaccharides from Antrodiacamphorata modulates dendritic cell function and alleviates allergeninduced T helper type 2 responses in a mouse model of asthma," Immunology, vol. 129, no. 3, pp. 351-362, 2010.

[21] O. Sukhbaatar, D. Kimura, M. Miyakoda et al., "Activation and IL-10 production of specific CD4+ T cells are regulated by IL-27 during chronic infection with Plasmodium chabaudi," Parasitology International, vol. 74, p. 101994, 2020.

[22] D. M. Shaw, F. Merien, and A. Braakhuis, "T-cells and their cytokine production: the anti-inflammatory and immunosuppressive effects of strenuous exercise," Cytokine, vol. 104, pp. 136-142, 2018. 


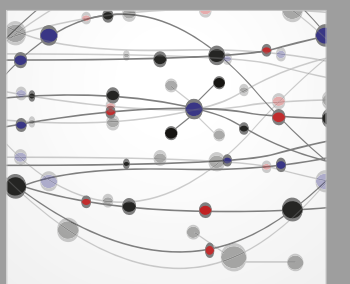

The Scientific World Journal
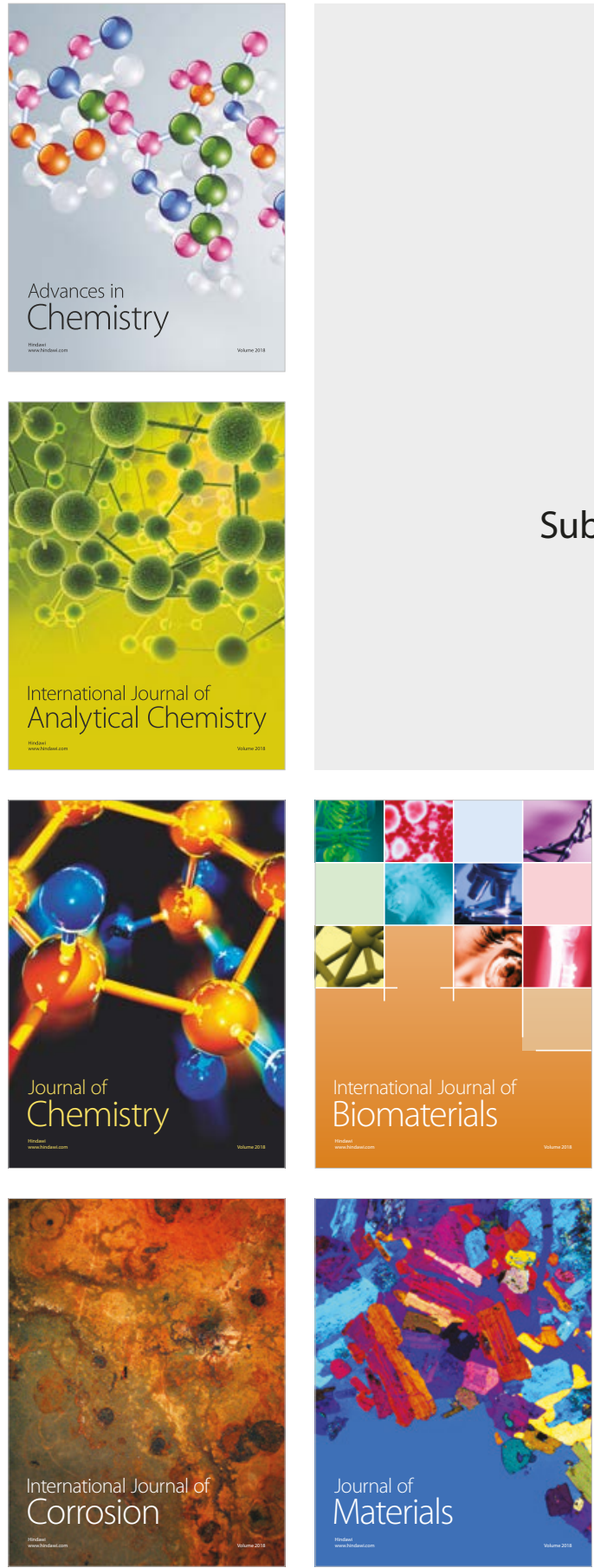

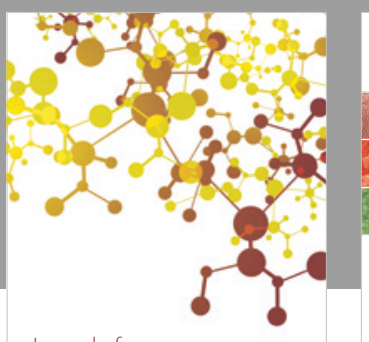

Journal of

Applied Chemistry
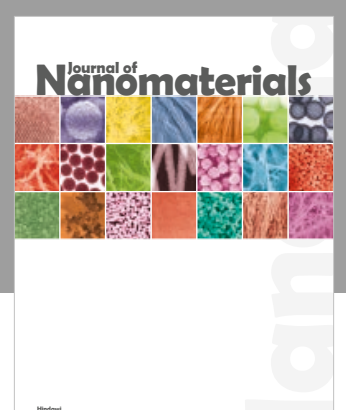

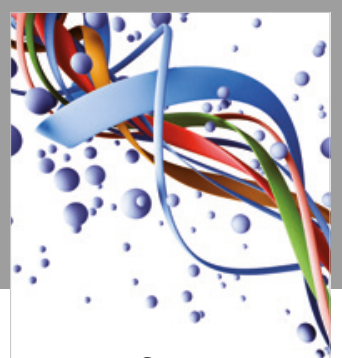

Scientifica

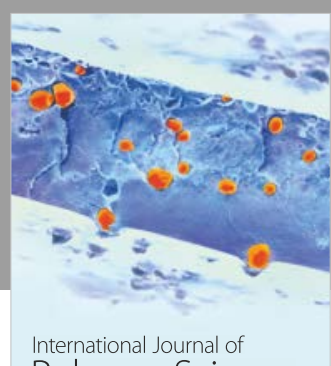

Polymer Science

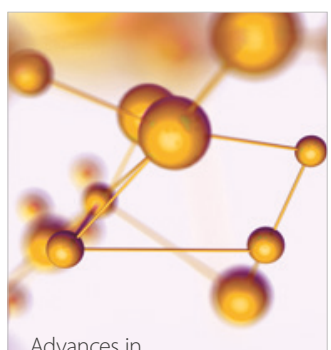

Physical Chemistry
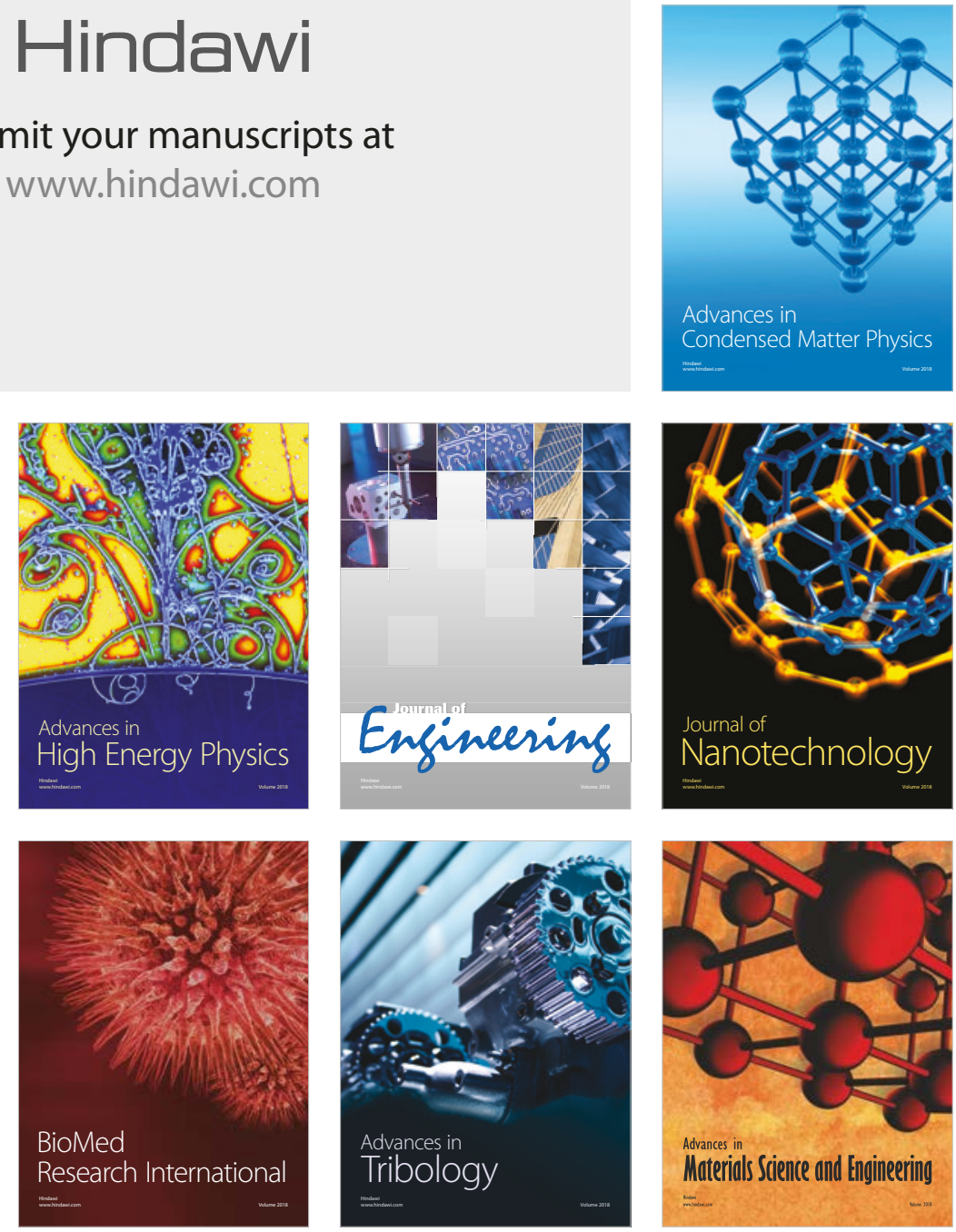